\title{
Inhibition of c-FLIP by RNAi Enhances Sensitivity of the Human Osteogenic Sarcoma Cell Line U2OS to TRAIL- Induced Apoptosis
}

\author{
Ya-Ping Zhang, Qing-Hong Kong, Ying Huang, Guan-Lin Wang*, Kwen-Jen Chang*
}

\begin{abstract}
To study effects of cellular FLICE (FADD-like IL-1ß-converting enzyme)-inhibitory protein (c-FLIP) inhibition by RNA interference (RNAi) on sensitivity of U2OS cells to tumor necrosis factor (TNF)-related apoptosis-inducing ligand (TRAIL)-induced apoptosis, plasmid pSUPER-c-FLIP-siRNA was constructed and then transfected into U2OS cells. A stable transfection cell clone U2OS/pSUPER-c-FLIP-siRNA was screened from the c-FLIP-siRNA transfected cells. RT-PCR and Western blotting were applied to measure the expression of c-FLIP at the levels of mRNA and protein. The results indicated that the expression of c-FLIP was significantly suppressed by the c-FLIP-siRNA in the cloned U2OS/pSUPER-c-FLIP-siRNA as compared with the control cells of U2OS/pSUPER. The cloned cell line of U2OS/pSUPER-c-FLIP-siRNA was further examined for TRAILinduced cell death and apoptosis in the presence of a pan-antagonist of inhibitor of apoptosis proteins (IAPs) AT406, with or without $4 \mathrm{hrs}$ pretreatment with rocaglamide, an inhibitor of c-FLIP biosynthesis, for $24 \mathrm{hrs}$. Cell death effects and apoptosis were measured by the methods of MTT assay with 3-(4,5-dimethylthiazol-2-yl)2,5-diphenyltetrazolium bromide and flow cytometry, respectively. The results indicated that TRAIL-induced cell death in U2OS/pSUPER-c-FLIP-siRNA was increased compared with control cells U2OS/pSUPER in the presence or absence of AT406. Flow cytometry indicated that TRAIL-induced cell death effects proceeded through cell apoptosis pathway. However, in the presence of rocaglamide, cell death or apoptotic effects of TRAIL were similar and profound in both cell lines, suggesting that the mechanism of action for both c-FLIP-siRNA and rocaglamide was identical. We conclude that the inhibition of c-FLIP by either c-FLIP-siRNA or rocaglamide can enhance the sensitivity of U2OS to TRAIL-induced apopotosis, suggesting that inhibition of c-FLIP is a good target for anti-cancer therapy.
\end{abstract}

Keywords: c-FLIP - RNA interference -TRAIL - osteogenic sarcoma - U2OS cells

Asian Pac J Cancer Prev, 16 (6), 2251-2256

\section{Introduction}

Osteosarcoma is the most common form of primary malignant bone tumor that mainly occurs in juvenile patients. It is a high-grade neoplasm with rapid growth and early metastasis (Marina et al., 2004). The mechanisms of formation and development of osteosarcoma have been studied for a long time. Recently, more evidences showed that c-FLIP plays important roles in regulating tumor growth (Safa, 2012).

TRAIL is a member of the structurally related TNF family and considered a promising anti-cancer drug (Wiley et al., 1995). To date, five TRAIL receptors have been identified: TRAIL-R1 (DR4), TRAIL-R2 (DR5), TRAIL-R3 (DcR1, TRID), TRAIL-R4 (DcR2, TRUNDD) and osteoprotegerin. Among these receptors, death receptors 4 and 5 (DR4 and DR5) are capable of recruiting adaptor proteins of Fas associated death domain
(FADD) to trigger downstream caspase-8/10 activation upon binding with TRAIL ligand (Carlo-Stella et al., 2007). Protection from death receptor (DR)-mediated apoptosis has been proposed as an important step in the development of malignancy. An important regulator of DR-induced death is c-FLIP. c-FLIP has homology to caspase- 8 and -10 but lacks of their protease activity and thereby prevents the activation of procaspase-8/10 and potently inhibits apoptosis mediated by DRs (Debatin and Krammer, 2004; Thorbum, 2004). As an important antiapoptotic protein, dysregulation of c-FLIP expression has been proven to be one of the major determinants of the resistance to death ligands such as FasL and TRAIL, suggesting that targeting c-FLIP is an appealing way to develop anticancer therapy (Djerbi et al., 1999; Micheau et al., 2002; Abedini et al., 2004). Moreover, studies suggest that down-regulation of c-FLIP could enhance TRAILmediated apoptosis (Woo et al., 2012). 
Nowadays, high-dose chemotherapy plus surgical treatment is used in the treatment of osteosarcoma but results in resistance to chemotherapy, side effects, and other problems that seriously influence the therapeutic effect (Soares et al., 2012).

RNAi can highly specifically silence targeted genes in mammalian cells using small interfering RNA (siRNA), and has been a powerful tool in studying the cell function of any gene (Brummelkamp et al., 2002). Also cancer is obviously an important target for siRNA-based therapies the most promising approach for a wide range of diseases (Mollaie et al., 2013). In this study, plasmid encoded siRNA against c-FLIP was constructed, and transfected into U2OS, a typical human osteogenic sarcoma cell line, to inhibit the c-FLIP expression. Our purpose in this study was to explore the function of c-FLIP on apoptosis of cancer cells, and to look for a new strategy to enhance the sensitivity of human osteogenic sarcoma to TRAIL or DR4/5 activation.

\section{Materials and Methods}

\section{Reagents}

AT406 (Selleckchem, USA), rocaglamide (Enzo Life Science, USA) and TRAIL (Peprotech, USA) were purchased. pSUPER. puro was purchased from Oligoengine (Madison St., WA, USA), Dulbecco's modified eagle's medium (DMEM) and fetal bovine serum (FBS) were purchased from HyClone (South Logan, UT, USA). c-FLIP-siRNA was purchased from Bioneer (Kogle 2, DK-2970 Hørsholm, Denmark), FuGENE ${ }^{\circledR}$ HD was purchased from Promega (Madison, WI, USA), puromycin was purchased from InvivoGen (Shatin, Hong Kong). Monoclonal antibody to c-FLIP (NF6) was purchased from Enzo Life Sciences (Madison Avenue, NY, USA), 3-(4,5-dimethylthiazol-2-yl)-2,5-diphenyltetrazolium bromide (MTT) was purchased from Solarbio (Beijing, China). Dimethyl sulfoxide (DMSO) was purchased from Sigma. Annexin V/FITC and Propidium Iodide Staining Solution (PI) were purchased from Becton Dickinson (San Jose, CA, USA).

\section{Cell lines and cell culture}

Human osteogenic sarcoma cell line U2OS was provided by Professor Ying Luo Laboratory at the Medical Faculty of Kunming University of Science and Technology. The cells were grown in DMEM medium (HyClone, USA) supplemented with 10\% FBS (HyClone, USA). The cells were maintained in a humidified $37^{\circ} \mathrm{C}$ incubator with $5 \% \mathrm{CO}_{2}$.

\section{Plasmid construction}

The pSUPER RNAi Vector (pSUPER. puro) was applied for construction. According to Nakajima et al. (2008), c-FLIP-siRNA used:

5' - GGAGCAGGGACAAGUUACA(dTdT)3'(sense); 5'-UGUAACUUGUCCCUGCUCC(dTdT)3'(antisense).

These above siRNA-encoding complementary single stranded oligonucleotides, were hybridized to give Xho Iand Hind III- compatible overhangs, and then ligated into
pSUPER. puro. The constructed plasmids were confirmed by sequencing and named as pSUPER-c-FLIP-siRNA.

\section{Transfection of cells}

Plasmids were transfected into cells using FuGENE ${ }^{\circledR}$ HD transfection reagent. In brief, one day prior to transfection, cells were seeded without antibiotics in 24-well plate at $4 \times 10^{4}$ cells/well, corresponding to a density of $70 \%-80 \%$ at the time of transfection. $0.55 \mu \mathrm{g}$ plasmids and $1.7 \mu \mathrm{l} \mathrm{FuGENE}{ }^{\circledR}$ HD per well were mixed with DMEM containing $10 \%$ FBS to total $25 \mu$ l. These reagents were combined and incubated for 10 minutes at room temperature before adding to the cells in DMEM containing $10 \%$ FBS. Cells were incubated at $37^{\circ} \mathrm{C}$ for 48 hrs. The cells with the transfection efficiencies above $50 \%$ examined on next study as judged form the fluorescence appearance co-transfected with a pCMV-AC-GFP vector (Origene, USA). Puromycin at $1 \mu \mathrm{g} / \mathrm{ml}$ was added to cells transfected with pSUPER-c-FLIP-siRNA and control plasmid for 5-6 days, then DMEM containing 20\% FBS was added into cells to be kept on cultivating. The stably transfected U2OS cell clones were cloned and collected, and named as U2OS/pSUPER-c-FLIP-siRNA and U2OS/ pSUPER as the control cells.

Semi-quantitative reverse transcription- $P C R$ ( $R T-P C R)$

Total cellular RNA was extracted from cells by RNAiso Plus (TaKaRa, Japan), $1 \mu$ g total RNA were reversed by M-MLV-Reverse Transcriptase (TaKaRa, Japan), the RT reaction was performed at $42^{\circ} \mathrm{C}$ for 60 minutes then $70^{\circ} \mathrm{C}$ for 15 minutes. $1 \mu$ l of total $\mathrm{RT}$ reaction volume was the template of the second DNA chain for the PCR. The primer sequences of Actin, as an internal control, were:5' -CTC CAT CCT GGC CTC GCT GT-3' (sense) and 5'-GCT GTC ACC TTC ACC GTT CC-3' (antisense), the annealing temperature was $54^{\circ} \mathrm{C}$ and the length of amplification was $268 \mathrm{bp}$. The primer sequences of c-FLIP ${ }_{\mathrm{L}}$ were 5'- GGC TCC CAG AGT GTG TAT GG-3' (sense) and 5'-AGC TTC TCG GTG AAC TGT GC-3' (antisense), the annealing temperature was $58^{\circ} \mathrm{C}$ and the length of amplification was $249 \mathrm{bp}$; The primer sequences of c-FLIP ${ }_{S}$ were 5'-GGA CCT TGT GGT TGA GTT GG-3' (sense), and 5'-ATC AGG ACA ATG GGC ATA GG-3' (antisense), the annealing temperature was $50^{\circ} \mathrm{C}$ and the length of amplification was $241 \mathrm{bp} .25 \mu \mathrm{l}$ total PCR products were separated by electrophoreses on $1.2 \%$ agarose gel containing ethidium bromide and visualized and photographed under UV light.

\section{Western blot analysis}

Cells were harvested after transfection and cloned. Whole cell proteins were isolated by using RIPA reagent (Beyotime, China). Protein concentration was determined by using a Protein Assay Kit (Bio-Rad, USA). Then an equal amount of total protein $(50 \mu \mathrm{g})$ from each lysate was loaded onto $10 \%$ sodium dodecyl sulfate-polyacrylamide gel electrophoresis (SDS-PAGE). After electrophoresis, separated proteins were transferred to polyvinylidene difluoride (PVDF) membranes (Millipore, USA) and blocked with TBS-T $(0.1 \mu \mathrm{mol} / \mathrm{L}$ Tris- $\mathrm{HCl}, \mathrm{PH} 7.5$; $0.9 \%$ sodium chloride; $0.05 \%$ Tween 20 ) containing $10 \%$ 
powdered non-fat milk for $2 \mathrm{hrs}$ at room temperature, and then incubated with mouse anti-human monoclonal antibodies against c-FLIP (Enzo, USA, 1:800 dilution), or GAPDH (Santa Cruz, USA, 1:2000 dilution) at $4^{\circ} \mathrm{C}$ overnight. After washing with TBS-T for $3 \times 10$ minutes, nitrocellulose membranes were incubated with 1:4000 dilution horseradish peroxidase (HBP)-labeled goat antimouse second antibody (Thermo, USA) for $2 \mathrm{hrs}$ at room temperature and washed with TBS-T for $3 \times 10$ minutes. Immunodetection was performed using SuperSignal West Femto Maximum Sensitivity Substrate (Millipore, USA).

\section{Cell viability analysis}

Cell viability was determined by MTT assay using 3-(4,5-dimethylthiazol-2-yl)-2,5-diphenyltetrazolium bromide (Li et al., 2014). Cells were seeded in 96-well plates in triplicate at a density of $5 \times 10^{3}$ cells per well with the complete culture medium. $180 \mu 1 \mathrm{DMEM}$ (containing $10 \%$ FBS) overnight, then treated with or without rocaglamide $(\mathrm{R}, 40 \mathrm{nM})$ for $4 \mathrm{hrs}$, and/or AT406 (A, $1 \mu \mathrm{g}$ / $\mathrm{ml}$ ) and TRAIL (T, 0, 1, 10, 50, $100 \mathrm{ng} / \mathrm{ml})$ for $24 \mathrm{hrs}$, and $50 \mu 1$ of MTT solution $(0.2 \mathrm{mg} / \mathrm{ml})$ was added into each well. After incubation at $37^{\circ} \mathrm{C}$ for $4 \mathrm{hrs}$, the MTT solution was removed and $150 \mu \mathrm{l}$ of DMSO was added to each well. The plate was incubated with DMSO at $27^{\circ} \mathrm{C}$ and shaken for 10 minutes to dissolve the formazan crystals. The formation of purple formazan crystals, which are proportional to the number of metabolically active viable cells, was determined by measuring the optical density at $490 \mathrm{~nm}$ wavelength on a microplate reader.

\section{Flow cytometry}

U2OS/pSUPER and U2OS/pSUPER-c-FLIPsiRNA cells were seeded at a density of $1 \times 10^{6}$ cells/ $\mathrm{ml}$ in 6-well plates and cultured for $24 \mathrm{hrs}$ in DMEM. After culturing, the cells were treated with AT406 (A, $1 \mu \mathrm{g} / \mathrm{ml})$ or TRAIL $(\mathrm{T}, 100 \mathrm{ng} / \mathrm{ml})$ or $\mathrm{A}+\mathrm{T}$ combination, respectively for $4 \mathrm{hrs}$ in a humidified atmosphere of $5 \% \mathrm{CO}_{2}$ at $37^{\circ} \mathrm{C}$. The percentage of apoptotic cells was detected by flow cytometry using an Annexin V/FITC and PI Apoptosis Detection Kit (Marczak et al., 2014). Cells were differentiated into viable (Q1-LL), dead (Q1-UL), early apoptotic (Q1-LR), and apoptotic cells (Q1-UR). Experiments were performed in triplicate.

\section{Statistical analysis}

Data are expressed as mean values \pm SD of at least three triplicate experiments. The statistical significance of differences between mean values was determined by factorial analysis. A $p$ value of less than 0.05 was considered to be significant. Statistical calculations were performed using SPSSv19 software (SPSS Inc., Chicago, IL, USA).

\section{Results}

Stable cell clones transfected with siRNA-encoding plasmids

Stable cell clones were screened out and were named as U2OS/pSUPER-c-FLIP-siRNA and the control clone as U2OS/pSUPER, respectively. The transfection efficiencies of the two clones were determined by fluoroscope for calculating the percent of GFP-expressed cells in all cells when they were co-transfected with a plasmid pCMV-ACGFP, and were both above 50\%. Both U2OS/pSUPER-cFLIP-siRNA and the control cells U2OS/pSUPER were grown for two weeks in the presence of puromycin $(1 \mu \mathrm{g} /$ $\mathrm{ml})$ and subsequently cloned to the cloned cell lines.

The mRNA expression of $c$-FLIP after stable transfection The mRNA expression was detected by RT-PCR (Figure 1). The c-FLIP mRNA of U2OS/pSUPER-c-FLIPsiRNA was inhibited markedly compared to control cells U2OS/pSUPER.

The protein expression of c-FLIP detected by Western blot after stable transfection

Western blot analysis (Figure 2) showed that the c-FLIP protein expression in clone cells U2OS/pSUPERc-FLIP-siRNA was decreased compared to control clone cells U2OS/pSUPER. The internal control GAPDH had no change in two cell lines.

\section{The cell viability after treatments}

Due to the cytotoxicity caused by rocaglamide alone (Figure $3 \mathrm{~B}$ ), the cell death effects induced by TRAIL were normalized as $100 \%$ in the absence of TRAIL (Figure 3A). As shown in Figure 3A and B, U2OS/ pSUPER-siRNA-c-FLIP cells and U2OS/pSUPER cells

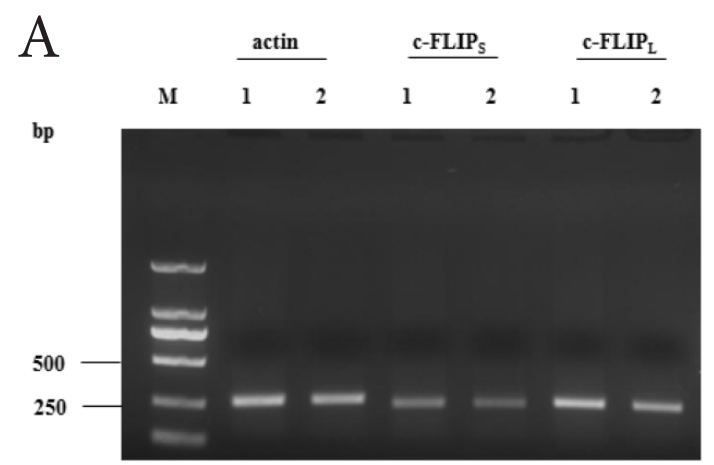

Figure 1. The mRNA of c-FLIP Down-regulation in U2OS/pSUPER-c-FLIP-siRNA Cells. A) $c$-FLIP and $_{S}$-FLIP mRNA expression in cells transfected with plasmids. Actin mRNA expression was used as internal reference. M: DL 2000bp Marker; 1: U2OS/pSUPER; 2: U2OS/pSUPER-c-FLIP-siRNA. B) Quantification of c-FLIP mRNA bands between U2OS/pSUPER and U2OS/pSUPER-c-FLIP-siRNA were achieved by computer-assisted densitometry using Image-J software. *Compared with U2OS/pSUPER cells $p<0.05$ 


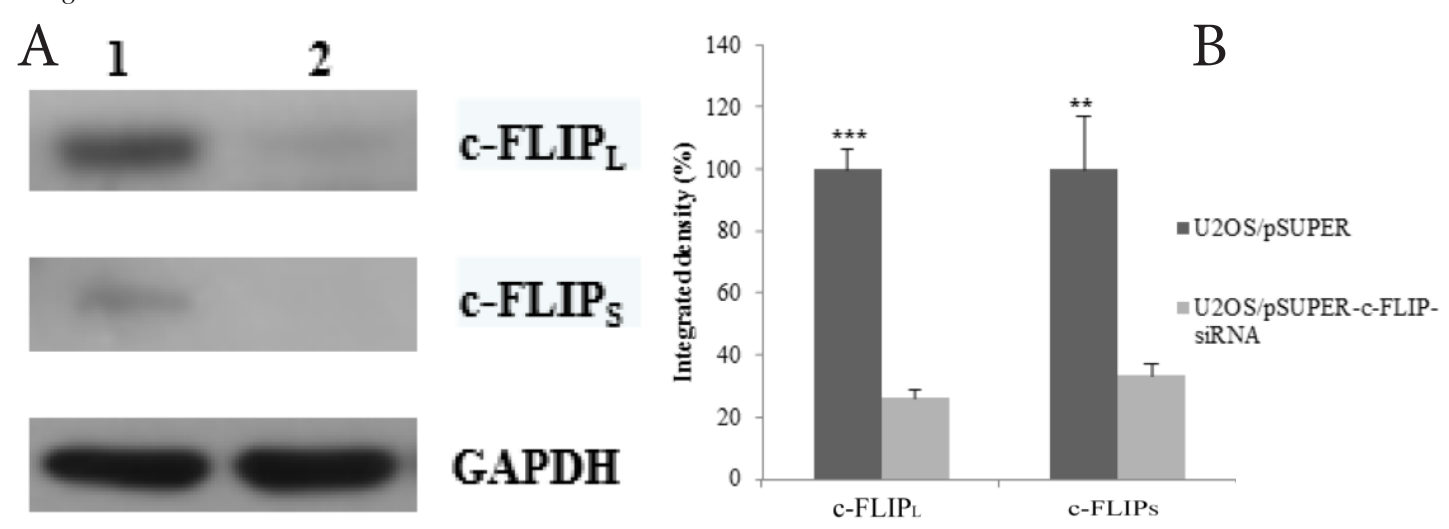

Figure 2. Expression of c-FLIP in Two Cell Lines Detected by Western Blot. A) Western blots of expressed proteins of c-FLIP ${ }_{L}$ and c-FLIP ${ }_{S}$. 1: U2OS/pSUPER; 2: U2OS/pSUPER-c-FLIP-siRNA. B) Relative density analysis on expressed proteins in Western blots of A. Quantifications of c-FLIP protein bands between U2OS/pSUPER and U2OS/pSUPER-c-FLIP-siRNA were achieved by computer-assisted densitometry using Image-J software. The diference bwteen these two cell lines are highly significant, $* * *$ Compared with U2OS/pSUPER cells $p<0.0001\left(\mathrm{c}-\mathrm{FLIP}_{\mathrm{L}}\right)$, **Compared with U2OS/pSUPER cells $p<0.01\left(\mathrm{c}-\right.$ FLIP $\left._{\mathrm{S}}\right)$
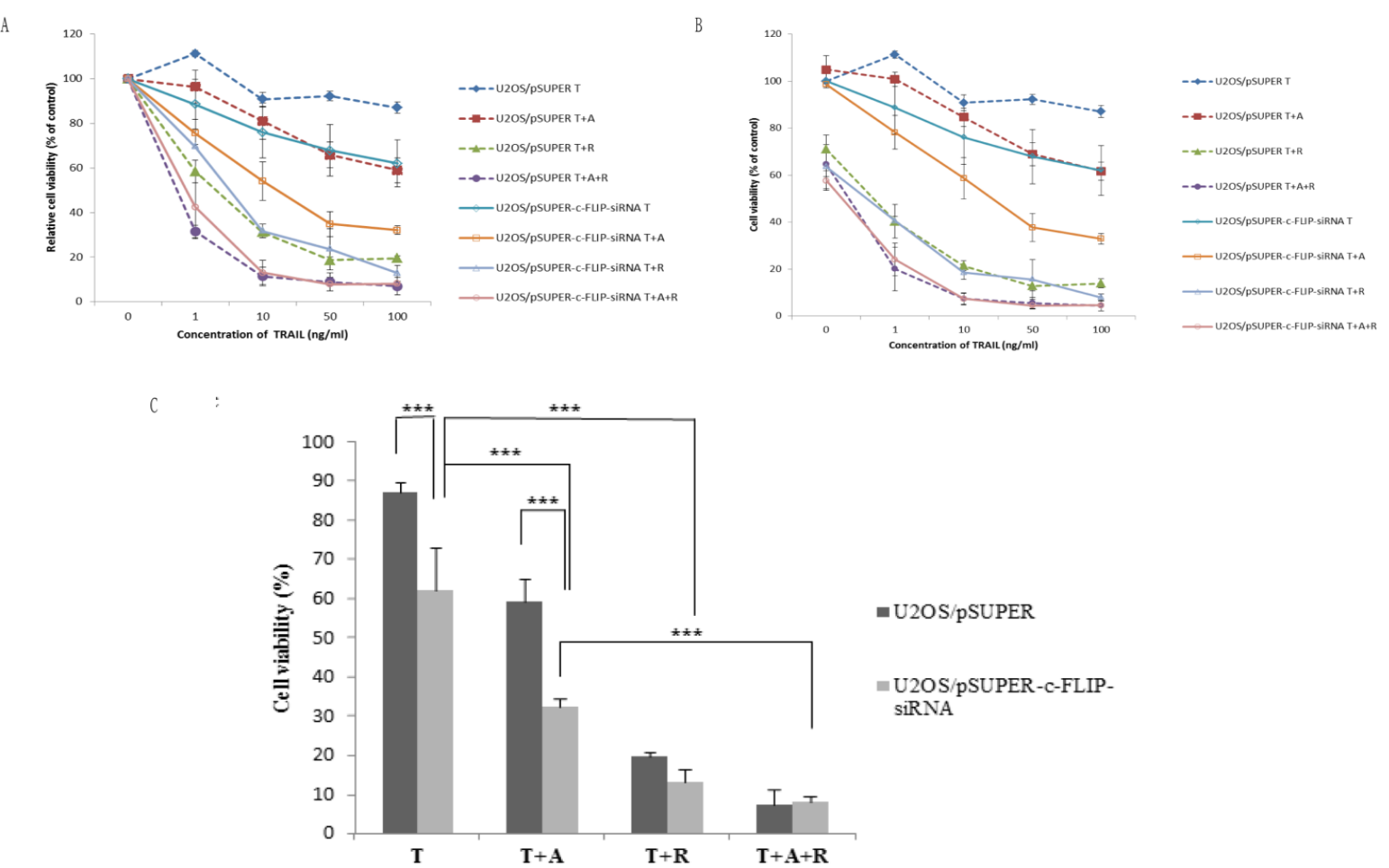

Figure 3. MTT Assay Detected The Cell Viabilities of U2OS/pSUPER-siRNA-c-FLIP Cells and U2OS/pSUPER Cells after Treatments of $\mathbf{T}, \mathbf{T}+\mathbf{A}, \mathbf{T}+\mathbf{R}$, and $\mathbf{T}+\mathbf{A}+\mathbf{R}$ Triple Combination. A) Due to some cytotoxicity caused by the treatment of rocaglamide, the cell viability detected by MTT assay after various treatments as indicated was normalized with the cell viability in the absence of TRAIL as 100\% viability. Data represent the mean \pm SD of at least three independent experiments. B) Un-normalized cell viability after various treatments as indicated were presented to show the cytotoxity caused by rocaglamide. The data represent the mean \pm SD of at least three independent experiments. C) Statistic analysis for above cell viability test after three separate experiments (TRAIL (T, 100ng/ml) and/or AT406 (A, $1 \mu \mathrm{g} / \mathrm{ml}$ ), and/or rocaglamide (R, 40nM)). ***Compared U2OS/ pSUPER cells. $p<0.0001$. The data represent the mean $\pm \mathrm{SD}$ of at least three independent experiments

were treated with TRAIL at the concentration indicated, and with or without AT406 $(1 \mu \mathrm{g} / \mathrm{ml})$. TRAIL produced more cell death of U2OS/pSUPER-siRNA-c-FLIP cells than control cells either in the absence or presence AT406, and the differences were significant (Figure 3C). However, the pretreatment of rocaglamide $(40 \mathrm{nM})$ for $4 \mathrm{hrs}$, and then with or without AT406 $(1 \mu \mathrm{g} / \mathrm{ml})$, TRAIL induced profound effects of cell death in both the U2OS/pSUPERsiRNA-c-FLIP cells and the control cells (Figure 3A and $\mathrm{B})$, and showed no differences between these two cell lines (Figure 3C).

\section{Flow cytometry detects cell apoptosis}

The effects of AT406, TRAIL or A+T combination on cell apoptosis were analyzed by flow cytometry. While AT406 alone had no apoptotic effect on both cell lines (Figure 4A and B), TRAIL induced more cell apoptosis of U2OS/pSUPER-siRNA-c-FLIP as compared with U2OS/ pSUPER (Figure 4A and B). TRAIL in combination with AT406 induced more cell apoptosis in both cell lines than either TRAIL or AT406 alone, and the apoptotic effects was greater in U2OS/pSUPER-siRNA-c-FLIP than in control cell line. $(p<0.05$; Figure $4 \mathrm{~A}$ and $\mathrm{B})$. 

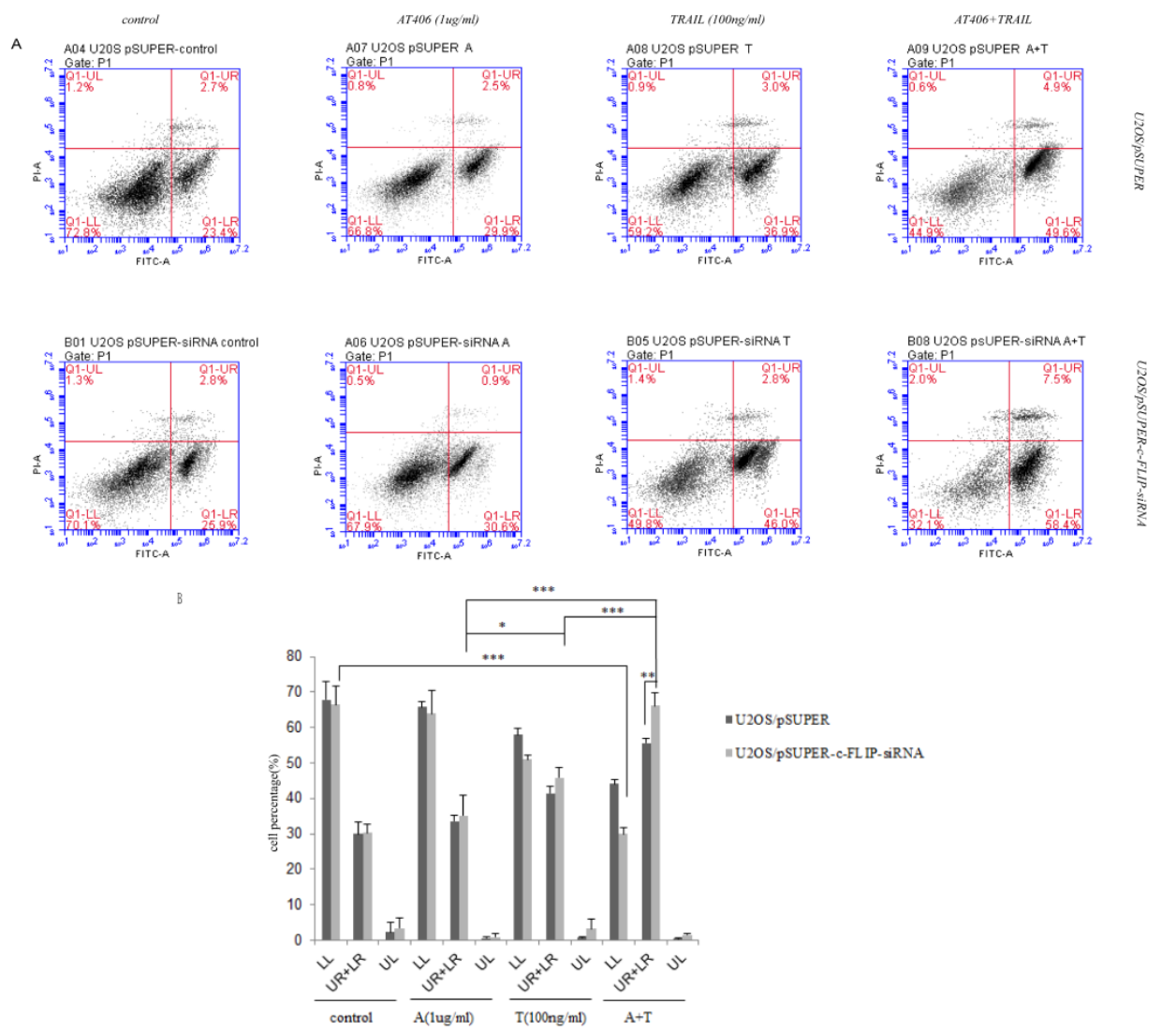

Figure 4. Effects of AT406 and TRAIL on The Apoptosis of U2OS/pSUPER-siRNA-c-FLIP Cells and U2OS/ pSUPER Cells. Cells were treated with AT406 $(1 \mu \mathrm{g} / \mathrm{ml})$ or TRAIL $(100 \mathrm{ng} / \mathrm{ml})$ alone and AT406 combined with TRAIL, respectively for $4 \mathrm{hrs.} \mathrm{A)} \mathrm{Cells} \mathrm{were} \mathrm{harvested} \mathrm{for} \mathrm{cell} \mathrm{apoptosis} \mathrm{analysis} \mathrm{by} \mathrm{flow} \mathrm{cytometry} 4 \mathrm{hrs}$ after drug treatment. B) Percentage of treated cells at different phases after various treatments. Data represent mean \pm SD from three independent experiments $(p<0.05)$

\section{Discussion}

A large number of previous studies found that a variety of tumors will be resistant to chemotherapy, radiation therapy and the effect of the TRAIL, this related to the expression of c-FLIP and clinical poor prognosis (Mezzanzanica et al., 2004; Valnet-Rabier et al., 2005; Gao et al., 2006; Ullenhag et al., 2007). RNAi technology, with its capability to knock down the expression of targeted genes (the vulnerable nodes), is moving into the clinic to target these nodes, which are integral to tumor maintenance, with a low risk of side-effects and to block intrinsic immunosuppressors thereby priming the tumor for immune attack (Rao et al., 2013).

The antisense oligonucleotides can be degraded rapidly in vivo and only have transient effects. This antisense oligonucleotides strategy has been restricted in both experimental and clinical applications (Iannitti et al., 2014). RNAi can highly specifically silence targeted genes in mammalian cells using siRNA, and has been a powerful tool in studying the function of any gene (Brummelkamp et al., 2002; Paddison et al., 2002). In this studying, two plasmids pSUPER-c-FLIP-targeted siRNA and pSUPER were constructed and transfected into U2OS, a cell line of osteosarcoma that is the most common, non-hematopoietic, primary malignant tumor of the bone occured predominantly in adolescents and young adults (Mirabello et al., 2009). Two stable clones were successfully screened out and named as U2SO/pSUPERc-FLIP-siRNA and U2SO/pSUPER. We found that there was no change of c-FLIP expression in U2OS/pSUPER the control cells, suggesting that the plasmid vectors had no harmful effects on biology of U2OS and could be applied safely. Mean while, the screened positive clone of U2OS/pSUPER-c-FLIP-siRNA showed the decreased expression of c-FLIP and had no effect on the expression of internal protein, indicating that the c-FLIP-targeted siRNA inhibited the expression of c-FLIP specifically.

After the treatments with TRAIL with or without AT406 for $24 \mathrm{hrs}$, the apoptosis rates of U2OS/pSUPERc-FLIP-siRNA were higher than those of control cells (Figure 3). The results support the conclusion that the decreased expression of c-FLIP in U2OS/pSUPER-cFLIP-siRNA has made the cells more sensitive to TRAILinduced apoptosis than U2OS/pSUPER, suggesting that TRAIL can better promote the association of procaspase- 8 to FADD in the decreased expression of c-FLIP in U2OS/ pSUPER-c-FLIP-siRNA cells and lead to increased apoptosis and cell death.

It is worth to note that rocaglamide, a translational inhibitor of c-FLIP biosynthesis (Zhu et al., 2009), in combination with AT406, a pan-antagonist of IAPs (Cai et al., 2011) and TRAIL (ART triple combination) produced similar profound cell apoptosis and cell death in both U2OS/pSUPER-c-FLIP-siRNA cells and the control U2OS/pSUPER cells (Figure 3A and B). The combination of rocaglamide with TRAIL also produced similar cell apoptosis and cell death in both cell lines, despite the slight less profound effects than ART triple combination (Fig $3 \mathrm{~A}$ and $\mathrm{B})$. These results suggest that the mechanisms of 
actions of c-FLIP-siRNA and rocaglamide in enhancing the sensitivity to TRAIL-induced apoptosis and cell death are very similar if not identical by the inhibition in the expression of c-FLIP.

It was reported that c-FLIP was overexpressed in human tumor cells, including colorectal carcinoma, hepatocellular carcinoma, pancreatic carcinoma, and prostate carcinoma (Hemandez et al., 2001; Elnemr et al., 2001; Okano et al., 2003; Zhang et al., 2004; Mori et al., 2005), and the c-FLIP overexpression indicated the tumors resistant to chemotherapy, radiation therapy, apoptotic effects of TRAIL and clinical poor prognosis (Mezzanzanica et al., 2004; Valnet-Rabier et al., 2005; Gao et al., 2006; Ullenhag et al., 2007), it prognosticates that c-FLIP may be an ideal target for enhancing TRAILsensitivity (Djerbi et al., 1999; Micheau et al., 2002; Abedini et al., 2004). Our results obtained from this study also confirmed this presume and provided a new way to raise the TRAIL-sensitivity of osteogenic sarcoma.

\section{Acknowledgements}

This study is supported by National Natural Science Foundation of China. The Grant number is 81360162 and 81260351 . The authors have no conflicts to disclose.

\section{References}

Abedini MR, Qiu Q, Yan X, et al (2004). Possible role of FLICKlike inhibitory protein (FLIP) in chemoresistant ovarian cancer cells in vitro. Oncogene, 23, 6997-7004.

Brummelkamp TR, Bernards R, Agami R (2002). A system for stable expression of short interfering RNAs in mammalian cells. Science, 296, 550-3.

Carlo-Stella C, Lavazza C, Locatelli A, et al (2007). Targeting TRAIL agonistic receptors for cancer therapy. Clin Cancer Res, 13, 2313-7.

Cai Q, Sun H, Peng Y, et al (2011). A potent and orally active antagonist (SM-406/AT-406) of multiple inhibitor of apoptosis proteins (IAPs) in clinical development for cancer treatment. J Med Chem, 54, 2714-26.

Djerbi M, Screpanti V, Catrina AI, et al (1999). The inhibitor of death receptor signaling, FLICE-inhibitory protein defines a new class of tumor progression factors. J Exp Med, 190, 1025-32.

Debatin KM, Krammer PH (2004). Death receptors in chemotherapy and cancer. Oncogene, 23, 2950-66.

Elnemr A, Ohta T, Yachie A, et al (2001). Human pancreatic cancer cells disable function of Fas receptors at several levels in fas signal transduction pathway. Int J Oncol, 18, 311-6.

Gao S, Wang H, Lee P, et al (2006). Androgen receptor and prostate apoptosis response factor- 4 target the c-FLIP gene to determine survival and apoptosis in the prostate gland. $J$ Mol Endocrinol, 36, 463-83.

Hernandez A, Wang QD, Schwartz SA, et al (2001). Sensitization of human colon cancer cells to TRAIL-mediated apoptosis. $J$ Gastrointest Surg, 5, 56-65.

Iannitti T, Morales-Medina JC, Palmieri B (2014). Phosphorothioate oligonucleotides: effectiveness and toxicity. Curr Drug Targets, 15, 663-73.

Li CL, Chang L, Guo L, et al (2014). $\beta$-elemene induces caspasedependent apoptosis in human glioma cells in vitro through the upregulation of bax and fas/ fasl and downregulation of bcl-2. Asian Pac J Cancer Prev, 15, 10407-12.

Micheau O, Thome M, Schneider P, et al (2002). The long form of FLIP is an activator of caspase- 8 at the fas death-inducing signaling complex. J Biol Chem, 277, 45162-71.

Mezzanzanica D, Balladore E, Turatti F, et al (2004). CD95mediated apoptosis is impaired at receptor level by cellular FLICE-inhibitory protein (long form) in wild-type p53 human ovarian carcinoma. Clin Cancer Res, 10, 5202-14.

Marina N, Gebhardt M, Teot L, et al (2004). Biology and therapeutic advances for pediatric osteosarcoma. Oncologist, 9, $422-4$.

Mori T, Doi R, Toyoda E, et al (2005). Regulation of the resistance to TRAIL-induced apoptosis as a new strategy for pancreatic cancer. Surgery, 138, 71-7.

Mirabello L, Troisi RJ, Savage SA (2009). Osteosarcoma incidence and survival rates from 1973 to 2004: data from the Surveilance, epidemiology, and end results program. Cancer, 115, 1531-43.

Mollaie HR, Monavari SH, Arabzadeh SA, et al (2013). RNAi and miRNA in viral infections and cancers. Asian Pac J Cancer Prev, 14, 7045-56.

Marczak A, Denel-Bobrowska M, Rogalska A, et al (2014). Cytotoxicity and induction of apoptosis by formamidinodoxorubicins in comparison to doxorubicin in human ovarian adenocarcinoma cells. Environ Toxicol Pharmacol, 39, 369-83.

Nakajima A, Kojima Y, Nakayama M, et al (2008). Downregulation of c-FLIP promotes caspase-dependent JNK activation and reactive oxygen species accumulation in tumor cells. Oncogene, 27, 76-84.

Okano H, Shiraki K, Inoue H, et al (2003). Cellular FLICK/ caspase-8-inhibitory protein as a principal regulator of cell death and survival in human hepatocellular carcinoma. Lab Invest, 83, 1033-43.

Paddison PJ, Caudy AA, Bernstein E, et al (2002). Short hairpin RNAs (shRNAs) induce sepuence-specific silencing in mammalian cells. Genes Dev, 16, 948-58.

Rao DD, Wang Z, Senzer N, Nemunaitis J (2013). RNA interference and personalized cancer therapy. Discov Med, 15, 101-10.

Safa AR (2012). c-FLIP, a master anti-apoptotic regulator. Exp Oncol, 34, 176-84.

Soares PI, Dias SJ, Novo CM, et al (2012). Doxorubicin vs. ladirubicin: methods for improving osteosarcoma treatment. Mini Rev Med Chem, 12, 1239-49.

Thorburn A (2004). Death receptor-induced cell killing. Cell Signal, 16, 139-44.

Ullenhag GJ, MukherjeeA, Watson NF, et al (2007). Overexpression of FLIP $_{L}$ is an independent marker of poor prognosis in colorectal cancer patients. Clin Cancer Res, 13, 5070-5.

Valnet-Rabier MB, Challier B, Thiebault S, et al (2005). c-FLIP protein expression in burkitt's lymphomas is associated with a poor clinical outcome [J]. Br J Haematol, 128, 767-73.

Wiley SR, Schooley K, Smolak PJ, et al (1995). Identification and characterization of a new member of the TNF family that induces apoptosis. Immunity, 3, 673-82.

Woo SM, Min KJ, Kwon TK (2012). Calyculin A causes sensitization to tumor necrosis factor-related apoptosisinducing ligand (TRAIL)-induced apoptosis by ROSmediated down-regulation of cellular FLICE-inhibiting protein (c-FLIP) and by enhancing death receptor 4 mRNA stabilization. Apoptosis, 17, 1223-34.

Zhang X, Jin TG, Yang H, et al (2004). Persistent c-FLIP(L) expression is necessary and sufficient to maintain resistance to tumor necrosis factor-related apoptosis-inducing ligandmediated apoptosis in prostate cancer. Cancer Res, 64 , 7086-91.

Zhu JY, Giaisi M, Kohler R, et al (2009). Rocaglamide sensitizes leukemic $\mathrm{T}$ cells to activation-induced cell death by differential regulation of CD95L and c-FLIP expression. Cell Death Differ, 16, 1289-99. 